\title{
UNA EXPERIENCIA DE GAMIFICACIÓN EN LA INTRODUCCIÓN A LA GESTIÓN EMPRESARIAL
}

\author{
Rey-Ares, Lucía'; Domínguez Feijoo, Gerardo²; \\ Crespo Pereira, Diego ${ }^{3} ;$ Ríos Prado, Rosa ${ }^{4}$ \\ ${ }^{1}$ Universidade da Coruña, Departamento de Empresa, \\ Facultade de Humanidades e Documentación, 0000-0002-5165-742X \\ ${ }^{2}$ Universidade da Coruña, Departamento de Empresa, Escola Universitaria de Deseño Industrial, \\ ${ }^{3}$ Universidade da Coruña, Departamento de Empresa, Escola Politécnica Superior, \\ 0000-0002-0797-249X \\ ${ }^{4}$ Universidade da Coruña, Departamento de Empresa, \\ Escola Politécnica Superior, 0000-0001-5755-7190
}

\section{RESUMEN}

La creación del Espacio Europeo de Educación Superior ha traído consigo la necesidad de crear y desarrollar metodologías docentes más activas e innovadoras, que convierten al alumnado en protagonista activo de su proceso de aprendizaje y apuestan por un aprendizaje comprensivo, centrado no solo en la adquisición de conocimientos, sino también de habilidades y competencias. Una de estas metodologías innovadoras es la gamificación, que consiste en la aplicación de juegos fuera de contextos lúdicos, como pueden ser los educativos, y que ha demostrado ser una herramienta eficaz en la motivación del alumnado universitario. Es por ello que este capítulo presenta una experiencia de gamificación, en particular centrada en la aplicación de un juego de rol en materias de introducción a la gestión empresarial. El juego consiste en formar diferentes grupos de estudiantes que constituirán empresas que competirán entre sí -convirtiendo así el aula en un mercado- y deberán tomar diferentes decisiones empresariales encaminadas a maximizar el beneficio empresarial. Los resultados de la experiencia revelan que ésta contribuye al refuerzo de los conocimientos teóricos desarrollados en el aula, a la mejora de competencias como el trabajo en equipo 0 la toma de decisiones, 0 a favorecer la motivación del alumnado.

PALABRAS CLAVE: juego de rol, toma de decisiones, negociación, enfoque centrado en el estudiante, innovación educativa. 


\section{CITA RECOMENDADA:}

Rey Ares, Lucía; Domínguez Feijoo, Gerardo; Crespo Pereira, Diego; Ríos Prado, Rosa (2021): Una experiencia de gamificación en la introducción a la gestión empresarial. En García Naya, J.A. (ed.) (2021). Contextos universitarios transformadores: a nova normalidade académica. Leccións aprendidas e retos de futuro. $V$ Xornadas de Innovación Docente. Cufie. Universidade da Coruña. A Coruña (pág. 295-304).

DOI capítulo: https://doi.org/10.17979/spudc.9788497498180.295

DOI libro: https://doi.org/10.17979/spudc. 9788497498180

\section{ABSTRACT}

The creation of the European Higher Education Area has brought about the need to create and develop more active and innovative teaching methodologies, which adopt a student-centred learning approach and are committed to comprehensive or holistic learning, thus not only focusing on the acquisition of knowledge, but also on the acquisition of skills and competences. One of these innovative methodologies is gamification, which consists of the application of game design elements outside their scope, such as learning environments, and which has proven to be an effective tool for motivating university students. This chapter presents a gamification experience, focused on the application of roleplaying in the courses of introduction to business management. The role-playing consists of dividing the class into different groups, constituting each group a different company. The companies created will compete with each other, thus turning the classroom into a marketplace, and they will have to make different business decisions aimed at maximizing business profits. The evidence of the experience reveals that it contributes to the reinforcement of the theoretical knowledge developed in the classroom, to the improvement of competences such as teamwork or decision-making, as well as to the increase of student motivation.

KEY WORDS: role playing, decision-making, negotiation, student-centred approach, educational innovation. 


\section{INTRODUCCIÓN}

La universidad, como institución al servicio del conocimiento, ha experimentado diferentes cambios desde su concepción, siendo uno de los más recientes y significativos la creación del Espacio Europeo de Educación Superior. En este contexto, el aprendizaje del alumnado a lo largo de la vida y la enseñanza centrada en éste para convertirlo en protagonista activo de su proceso de aprendizaje se convierten en objetivos clave en la educación superior (RodicioGarcía et al., 2021). Para alcanzar estos objetivos, han surgido diferentes metodologías docentes, más activas e innovadoras, entre las que se encuentra la gamificación; metodologías que dejan atrás el aprendizaje memorístico para centrarse en un aprendizaje comprensivo, donde el alumnado adquiere conocimientos, pero también competencias y habilidades.

El uso de juegos o elementos de juegos en el ámbito educativo no es una cuestión novedosa; de hecho, en la década de los sesenta Piaget (1962) ya señalaba que los juegos pueden ser de gran interés en el ámbito de la educación infantil. Desde entonces, diferentes publicaciones han confirmado que la gamificación, entendida como el "uso de elementos de diseño de juegos en contextos no lúdicos" (Deterding et al., 2011), constituye una herramienta útil a la hora de favorecer el aprendizaje, la motivación y el compromiso del alumnado (Barbosa y Sorto, 2016; Martí-Parreño et al., 2016).

En lo que sigue de este capítulo presentaremos una experiencia de gamificación llevada a cabo por profesorado del Grupo de Innovación Docente en Organización de Empresas (InnovaOrg) de la Universidade da Coruña, en asignaturas de primer curso. En particular, la experiencia se ha llevado a cabo en las asignaturas Gestión Empresarial, impartida en los Grados en Ingeniería Mecánica e Ingeniería en Tecnologías Industriales, y Fundamentos de Gestión de las Empresas de Moda, impartida en el Grado en Gestión Industrial de Moda, siendo un total de 69 estudiantes quienes han formado parte de ésta. 
El juego de rol que se ha diseñado trata de acercar al alumnado a las operaciones básicas de gestión empresarial mediante la toma de decisiones en un contexto de competencia y próximo a la realidad, tratando así, de una forma lúdica, de mejorar los conocimientos y competencias del alumnado, buscando a su vez aumentar su motivación e interés en la asignatura. Para ello, el aula se convierte en un mercado formado por diferentes empresas (o grupos de estudiantes) que compiten entre sí buscando obtener el máximo beneficio y cuota de mercado.

Los resultados de esta experiencia demuestran que la gamificación constituye una herramienta eficaz en la mejora de competencias del alumnado, a la vez que contribuye al refuerzo de los conocimientos teóricos adquiridos en el aula y a aumentar la motivación del alumnado.

\section{OBJETIVOS}

La realización de este juego de rol, como se ha referido previamente, busca acercar al alumnado que tiene un primer contacto con la gestión empresarial a la toma de decisiones en este ámbito desde un enfoque más lúdico y dinámico.

En concreto, los objetivos de carácter general que se persiguen con este juego son fundamentalmente tres: (a) el refuerzo y aplicación práctica de los contenidos teóricos desarrollados en el aula; (b) el desarrollo y mejora de diferentes competencias de carácter transversal, como la de gestión del tiempo y la información, el trabajo en equipo, la toma de decisiones en contextos de incertidumbre o la negociación; y (c) el aumento de la motivación e interés del alumnado en la asignatura; cuestión de especial importancia en todos los cursos académicos, pero especialmente en el curso 2020/21, dado el contexto de desánimo general derivado de la situación de pandemia que atravesamos.

Los objetivos específicos que se persiguen con esta experiencia, más vinculados con la aplicación práctica de los contenidos estudiados en el aula, buscan que el alumnado: (a) mejore la capacidad de análisis de datos y toma de decisiones, usando y aplicando para ello herramientas y técnicas que se han visto en el aula, como el análisis del umbral de rentabilidad 
0 el cálculo de los beneficios empresariales; y (b) trate de maximizar los beneficios en su empresa.

\section{DESCRIPCIÓN DE LA EXPERIENCIA}

El juego de rol que hemos realizado comienza con la creación de tres grupos en cada una de las clases interactivas; grupos que pueden incluir tanto a estudiantes que asisten a la sesión interactiva de forma presencial como a estudiantes que asisten de forma telemática. En este sentido, aunque la docencia de las dos asignaturas en las que se ha llevado a cabo esta experiencia es presencial, hay alumnado que puntualmente ha seguido la docencia de forma telemática, por encontrarse, por ejemplo, en situaciones de cuarentena preventiva.

Cada uno de los tres grupos formados en el aula constituirá una empresa, de forma que las tres empresas (o grupos de estudiantes) formarán un mercado donde cada una de ellas competirá por alcanzar la mayor cuota de mercado y beneficios empresariales. Una vez creados los grupos, cada uno de ellos recibe un documento con las instrucciones específicas que detallan cómo va a transcurrir el juego; instrucciones que también se explican en el aula.

El juego de rol que presentamos se ha adaptado a cada una de las titulaciones donde se ha aplicado, de forma que aspectos como el tipo de producto, los costes, los niveles de demanda o la segmentación del mercado pueden variar para cada una de las asignaturas; motivo éste por el cual no se detallarán en este capítulo.

Con independencia de estas particularidades, las instrucciones generales establecen que la práctica consiste en un juego donde, en diferentes turnos o rondas, cada grupo deberá tomar tres decisiones principales, relativas al establecimiento de:

- Precio de venta del producto que comercializa, asumiendo que todas las empresas venderán el mismo tipo de producto.

- Nivel de calidad del producto vendido, que podrá variar en un rango entre 0 y 10 , siendo 0 el nivel mínimo de calidad. El alumnado debe ser consciente de que los clientes tienen la capacidad de recordar los niveles de calidad previos que ofrece cada 
empresa, de forma que de un turno a otro, los consumidores son capaces de recordar las calidades anteriores y tomar su decisión de compra en función de dichos valores.

- Capacidad de producción de cada empresa. Cada empresa parte de la misma capacidad en cuanto a número de unidades fabricadas (cifra que se detalla en las instrucciones), pero tiene la posibilidad de modificar la capacidad inicialmente establecida en las sucesivas rondas del juego.

Las instrucciones detallan los costes del producto, tanto fijos como variables, especificando cómo varían los costes en función del nivel de calidad del producto, en tanto que, a mayor nivel de calidad, mayores serán los costes.

El alumnado no conoce con exactitud la demanda, pero se le informa de los niveles entre los que la demanda puede variar; variación que se producirá de forma aleatoria. Igualmente, se le informa de que el mercado se divide en dos segmentos principales de consumidores: uno de los segmentos toma sus decisiones guiado fundamentalmente por la calidad del producto, mientras que otro de los segmentos toma sus decisiones de compra guiado fundamentalmente por el precio. Se precisa el porcentaje que representa cada segmento y se señala que todas las empresas deberán seguir una estrategia de marketing indiferenciado, de forma que deberán establecer un único precio y nivel de calidad en cada una de las rondas del juego.

Explicadas las instrucciones en el aula, se establece un tiempo máximo para que cada grupo tome las decisiones antes indicadas, y cubra una plantilla. Para ello, cada equipo debe nombrar a un representante, que será quien se encargue de cubrir la plantilla y entregársela al profesorado. Una vez el profesorado recibe la plantilla, ha de introducir los datos en un archivo Excel donde se ha diseñado previamente un modelo de reparto o utilidad a partir de un modelo logit binomial que tiene como variables principales precio y coste.

Introducidos los datos en el archivo Excel, se obtienen diferentes resultados, como son el volumen de ventas de cada grupo en número de unidades, la cuota de mercado, los beneficios de cada grupo 0 empresa en el turno actual y los beneficios acumulados. El dato referido al 
volumen de ventas de cada empresa se comunica de forma individual a cada grupo, para que pueda hacer sus cálculos de cara a la siguiente ronda, mientras que los datos relativos a la cuota de mercado (esto es, porcentaje de las ventas que corresponde a cada empresa), y a la evolución de los precios y calidades de las tres empresas se muestran de forma conjunta a todos los equipos. Cada equipo deberá calcular los beneficios que va obteniendo.

Comunicados los resultados de la primera ronda, se da inicio a la segunda ronda, dando un tiempo máximo para que cada equipo tome sus decisiones. En caso de que algún equipo no comunique sus resultados en el tiempo máximo que se establece, se le asignarán unos datos por defecto, que se especifican en la plantilla de instrucciones al inicio del juego.

Se realizan tantas rondas como el tiempo de cada sesión interactiva permita y, al finalizar las diferentes rondas, se muestra al alumnado los beneficios que cada empresa ha obtenido, así como los beneficios totales ( 0 acumulados) tras las sucesivas rondas de negociación, resultando "vencedora" aquella empresa que consigue obtener mayores beneficios.

\section{RESULTADOS}

Tras la puesta en práctica de esta experiencia se ha valorado en qué medida ha contribuido a la consecución de los objetivos planteados. Para ello, además de analizar los resultados que cada uno de los grupos obtienen en el juego, se ha realizado una encuesta de satisfacción entre el alumnado participante en la experiencia.

En términos generales, la valoración que el alumnado realiza de la experiencia es muy satisfactoria. Así, en relación con el primer objetivo general que plantemos, un 91\% del alumnado afirma que esta práctica le ha permitido entender mejor la materia y los diferentes conceptos teóricos estudiados en el aula. En este sentido, el alumnado afirma que la práctica le ha permitido aproximarse a las estrategias competitivas que toman las empresas en el mundo real; conocer cómo los resultados de una empresa están condicionados no solo por sus propias decisiones, sino por las que tomen las empresas competidoras; 0 darse cuenta de 
que el nivel de beneficios es una variable clave, que muchas veces desatienden en la toma de decisiones, guiados por el deseo de conseguir la máxima cuota de mercado.

En lo que a la evaluación de las competencias se refiere, como refleja la Figura 1, en una escala Likert 1-5, donde el valor 5 refleja el mayor grado de acuerdo, un 85,7\% del alumnado manifiesta estar de acuerdo o muy de acuerdo en que el juego de rol ha contribuido al desarrollo de las competencias de trabajo en equipo y cooperación, un 92,9\% del alumnado está de acuerdo o muy de acuerdo en que contribuye a la mejora de la capacidad de análisis, diagnóstico y toma de decisiones; y de forma similar, un $92,8 \%$ considera que el juego de rol llevado a cabo en el aula contribuye a mejorar su aprendizaje.

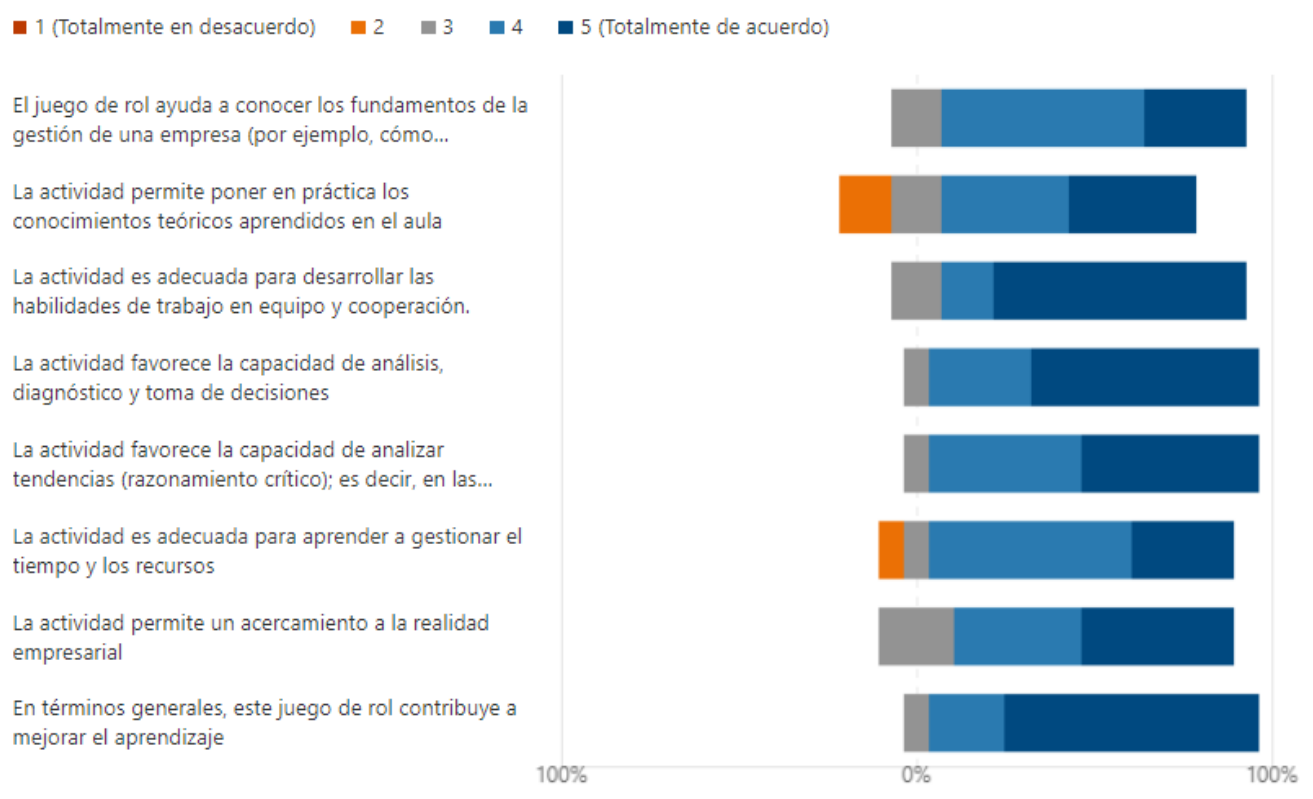

Figura 13. Valoración de competencias por parte del alumnado participante (escala Likert 1-5)

Si bien, hemos constado que no todo el alumnado pone en práctica herramientas como el análisis del umbral de rentabilidad o cálculo del punto muerto. En ocasiones, el alumnado se deja guiar por su instinto y no aplica estas herramientas, o no lo hace en todas las rondas del juego, lo que puede derivar en la obtención de resultados económicos poco favorables. 
El 100\% del alumnado coincide en señalar que esta práctica debería repetirse en el futuro, y que este tipo de actividades deberían usarse con más frecuencia si la asignatura lo permite. Es evidente, por tanto, que la experiencia de gamificación que hemos planteado ha contribuido en gran medida al logro de los objetivos planteados, así como al aumento de la motivación e interés del alumnado en las asignaturas donde se ha llevado a cabo esta experiencia.

\section{CONCLUSIONES}

La experiencia de gamificación que hemos puesto en práctica en las asignaturas de introducción a la gestión empresarial ha conseguido unos resultados altamente satisfactorios, como así se constata en la sección anterior. La experiencia ha permitido una aplicación práctica de los conceptos teóricos vistos en el aula; ha contribuido a la mejora de competencias como el trabajo en equipo, la gestión del tiempo y la información, o la toma de decisiones a través de la asunción de roles en un contexto que trata de simular la realidad; y ha fomentado la competencia entre el alumnado; competencia que, en algunos casos, ha sido excesiva, llegando incluso a derivar en pequeños conflictos entre el alumnado. Situación que no es más que un reflejo de la realidad y la competencia feroz que en algunos casos llegan a desarrollar entre sí las empresas.

Las principales dificultades que el alumnado ha señalado están condicionadas por las restricciones derivadas de las medidas sanitarias impuestas para hacer frente a la pandemia en las aulas universitarias. Una gran parte del alumnado considera que la necesidad de guardar la distancia social, de alguna forma, les ha dificultado interactuar y, por tanto, trabajar en equipo de forma eficiente. En este sentido, aunque el alumnado podía utilizar los canales disponibles en el equipo de Teams de las dos asignaturas para la comunicación dentro de cada grupo (de forma obligatoria en el caso de que alguna persona del grupo siguiese la clase de forma telemática), la comunicación demostró, en algunos casos, no ser tan fluida como en un contexto de normalidad.

De cara a la mejora de la actividad, en base al análisis de los resultados obtenidos y a las valoraciones obtenidas en la encuesta de satisfacción, creemos que sería necesario conceder 
más tiempo a cada grupo en la primera ronda del juego, porque los 15 minutos que se conceden parecen insuficientes. También creemos necesario insistir sobre la necesidad de que cada empresa debe centrarse en maximizar sus beneficios y no tanto su cuota de mercado. El análisis de los resultados deja entrever que, en algunos casos (los menos, afortunadamente) el alumnado ha actuado guiado por el deseo de obtener la mayor cuota de mercado, lo que lo ha llevado a tomar decisiones ilógicas como la fijación de un precio de mercado inferior al coste que, si bien puede llevarle a incrementar la cuota de mercado de forma sustancial, acabará por derivar en importantes pérdidas económicas, con consecuencias nefastas para la empresa.

\section{REFERENCIAS}

Barbosa, A. \& Soto, J.G. (2016). Gamification Jam Galicia. Difusión de la gamificación y formación del profesorado. En C. Monge \& P. Gómez (Eds.), II Congreso Virtual Iberoamericano sobre Recursos Educativos Innovadores CIREI (pp. 250-257). Alcalá de Henares: Universidad de Alcalá.

Deterding, S., Dixon, D., Khaled, R. \& Nacke, L. (2011). From game design elements to gamefulness: defining "gamification". En Proceedings of the $15^{\text {th }}$ International Academic MindTrek Conference: Envisioning Future Media Environments (pp. 9-15). ACM. https://doi.org/10.1145/2181037.2181040

Martí-Parreño, J., Méndez-lbáñez, E. \& Alonso Arroyo, A. (2016). The use of gamification in education: a bibliometric and text mining analysis. Journal of Computer Assisted Learning, 32(6), pp. 663-676. https://doi.org/10.1111/jcal.12161

Piaget, J. (1962). Play, dreams and imitation in childhood. Nueva York: W.W. Norton \& Co.

Rodicio-García, M.L., Ríos-de-Deus, M.P., Mosquera-González, M.J., Rego-Agraso, L. \& Penado, M. (2021). Mentoría en la Universidad: Formación de las Figuras Implicadas (MUFFIM). Módulo 1, El Espacio Europeo de Educación Superior como escenario para la mentoría. http://hdl.handle.net/2183/27499 